\title{
EFFECT OF MELATONIN ON REGENERATION OF CORTICAL NEURONS IN RATS WITH TRAUMATIC BRAIN INJURY
}

\author{
VOL 43 NO 4 DECEMBER 2020
}

\author{
Jianbin Ge, $M D^{1}$, Dandan Chen, $M D^{1}$, Jingjing Ben, $M D^{1}$, Xinjian Song, $M D^{2}$, Linqing Zou, $\mathrm{PhD}^{3}$, $\mathrm{Xin} Y \mathrm{Y}, \mathrm{PhD}^{3}$ \\ 1 Department of Pharmacy, the Second People's Hospital of Nantong, Nantong, China \\ 2 Department of Rehabilitation Medicine, the Second People's Hospital of Nantong, Nantong, China \\ 3 Department of Human Anatomy, Medical College, Nantong University, Nantong, China \\ Submitted 28th May 2020, Accepted 24th September 2020 \\ Clin Invest Med. 2020;43(4):E8-16. DOI: 10.25011/cim.v43i4.34829
}

\begin{abstract}
Purpose: To investigate the effect of melatonin on regeneration of cortical neurons in rats with traumatic brain injury (TBI).

Methods: Sprague-Dawley rats ( $n=36$ ) were randomly divided into sham, $T B \mid+v e h i c l e ~ a n d ~ T B I+$ melatonin groups. Cerebral blood flow and cognitive function were observed via laser Doppler flowmetry and by Morris water maze testing, respectively. The serum malondialdehyde (MDA) and superoxide dismutase (SOD) levels were used to assess oxidative stress. Immunofluorescence and terminal deoxynucleotidyl transferase dUTP nick end labelling assay was used to observe the newborn neurons and apoptotic cells.

Results: Cerebral blood flow in the TBI+melatonin group was higher than that of the TBI+vehicle group at one, 12,24 and $48 \mathrm{~h}$ post-injury, but the difference was not statistically significant $(P>0.05)$. The cognitive function of the rats was better in the TBI+melatonin group than the TBI+vehicle group $(P<0.05)$. The MDA and SOD levels were better in the TBI+melatonin group than the TBI+vehicle group $(P<0.05)$ and it was similar to the sham group at $14 \mathrm{~d}(P>0.05)$. The number of apoptotic cells was lower in the TBI+melatonin group than the TBI+vehicle group $(P<0.05)$. The number of $\mathrm{DCX}+/ \mathrm{BrdU}+$ immature newborn neurons and $\mathrm{NeuN}+/ \mathrm{BrdU}+$ mature newborn neurons was higher in the TBI+melatonin group than the TBI+vehicle group $(P<0.05)$.

Conclusion: These results indicated that melatonin reduced oxidative stress level and cells apoptosis of brain and promoted the regeneration of neurons in the cortex of rats with TBI, leading to improve cognitive functional recovery.
\end{abstract}

Correspondence to: Xin Yi, ntuyixin@foxmail.com

\section{INTRODUCTION}

The incidence of traumatic brain injury (TBI) is increasing in both developing and developed countries. Although the understanding of the pathophysiology of TBI has gradually improved, the prognosis of TBI patients is still poor, placing a heavy burden on families and society [13]. Brain injury causes a series of biochemical events in the cells of the injured area, including free radical-mediated injury, excitotoxicity and inflammation, anaerobic metabolism, intracellular calcium accumulation, mitochondrial dysfunction and diffuse axonal injury. These events lead to extensive and long-term apoptosis [4-6]. Virtually every cell is affected by this cascade of injury-induced biochemical events, but they do not respond in the same way. Neurons are the cells that are most sensitive to injury, and they also show selective vulnera- bility $[7,8]$.

Neural stem cells (NSCS) have become the focus of research because of a lack of treatments that effectively repair damaged brain tissue, prompting researchers to focus on potential ways to replace these lost cells. Previous studies showed that TBI can stimulate proliferation of endogenous NSCs but the activated NSCS fail to become mature neurons with in the injured cortex $[9,10]$. Therefore, promotion of the differentiation of proliferating NSCs into mature neurons is of great significance.

Melatonin ( $\mathrm{N}$-acetyl-5-methoxytryptamine) is a potent free radical scavenger, as well as an indirect antioxidant. Melatonin can remove singlet oxygen and hydroperoxide, and superoxide anion, hydroxyl and lipid peroxide radicals [11,12]. Melatonin acts as an indirect antioxidant 
by activating the most important antioxidant enzymesincluding superoxide dismutase (SOD), catalase and glutathione peroxidase-and improving mitochondrial electron transport efficiency [13,14]. Melatonin represents an attractive option as a neuroprotective approach in brain injury because of its potential efficacy and low toxicity. Previous studies showed a significant decrease in infarcted volume, protection of cells and improved outcome on neurobehavioral tests after administration of melatonin after ischemic brain injury in a rat model $[15,16]$. The role of melatonin on TBI has been studied too [17-20]; however, little is known about whether the administration of melatonin could promote the regeneration of neurons in rats with TBI. This study aims to investigate the effect of melatonin on regeneration of cortical neurons in rats with TBI.

\section{MATERIAL AND METHODS}

\section{Animal and group}

Animal procedures were carried out according to the National Institutes of Health Guidelines for the Care and Use of Laboratory Animals and approved by the Animal Experimental Committee of the Nantong University. Thirty-six Sprague-Dawley rats (220-250 g, both male and female) for the original study cohort were randomly assigned to three groups: sham (male/female: 6/6), TBI+vehicle (male/female: 6/6) and TBI+melatonin (male/ female: 6/6). Thirty-six Sprague-Dawley rats (220-250 g, both male and female) for the second study cohort were randomly assigned to three groups: sham group (male/ female: 6/6), TBI+vehicle group (male/female: 6/6) and $\mathrm{TB}+$ +melatonin (male/female: 6/6) group.

\section{Animal model of traumatic brain injury}

The controlled cortical impact (CCl) adult rat model of medium TBI was prepared according to the previous study [9]. After the rats were anesthetized with $2 \%$ pentobarbital sodium $(0.30 \mathrm{ml} / 100 \mathrm{~g})$, the rats were fixed to a stereotactic device. Under sterile conditions, a 4 $\mathrm{mm}$ diameter circular window (centered at antero-posterior $-3.5 \mathrm{~mm}$, medio-lateral $2.5 \mathrm{~mm}$ with reference to the bregma) was created in the parietal bone of the rat, taking care to avoid damaging the meninges. After the circular window was made, a pneumatic impact device (model FP302, AmScien Instruments LLC, USA) was immediately used to impact the rat cortex (air pressure was $1.5 \mathrm{~atm}, 1 \mathrm{~atm}=101.325 \mathrm{kPa}$ ). After the traumatic brain injury was successfully prepared, the bone window was immediately sealed with bone wax, and the rat skin incision was sutured layer by layer. Sham rats were subjected to all aspects of the protocol (anesthesia, skin incision and craniotomy) except for cortical impact. After recovery from anesthesia, the rats were returned to their cages according to group and gender with postoperative care and access to food and water ad libitum. There were no premature deaths in the rats during the preparation of the TBI model.

\section{Administration of melatonin}

Melatonin ( $6 \mathrm{mg} / \mathrm{ml}$ ) (Sigma, USA) was dissolved in normal saline containing $4 \%$ ethanol. Rats in the TBI+melatonin group were injected with melatonin $(15 \mathrm{mg} / \mathrm{kg})$ at $0 \mathrm{~h}, 24 \mathrm{~h}$ and $48 \mathrm{~h}$ post-injury by tail vein. Rats of sham and $\mathrm{TB} \mid+v e h i c l e$ groups were injected with equal dose of vehicle by tail vein.

\section{5-Bromo-2'-deoxyuridine injection}

All rats were intraperitoneally injected with 5-bromo-2'-bromodeoxyuridine (BrdU) solution (BrdU dissolved in normal saline, $10 \mathrm{mg} / \mathrm{ml}$ ) $(50 \mathrm{mg} / \mathrm{kg}$ ) (Sigma, USA) at zero, 24,48 and $72 \mathrm{~h}$ post-injury to label newborn cells.

\section{Laser Doppler flowmetry measurement}

The cerebral blood flow (CBF) was observed via laser Doppler flowmetry (LDF) as previously described by Rafols [21]. Briefly, LDF measurements were performed with a $3 \mathrm{~mm}$ in thickness and $10 \mathrm{~mm}$ in diameter LDF probe (Vasamedics, USA) at one, 12, 24 and $48 \mathrm{~h}$ post-injury. The Laserflo BPM2 (Vasamedics) was used for LDF recordings. A plate was attached to the skull with miniature anchor screws and a hole was drilled for the laser probe. On the plastic plate, a guiding cannula (Becton-Dickinson, USA) was positioned. The dental cement was used to fix the plate and cannula. The raw data from the Doppler probe were transferred to an A/D converter (DT21-EZ, USA) and stored in the computer using software (Labtech Notebook, Laboratory Technology, USA) for analysis. The 3,600 point moving average technique was used to analyze the raw data [22]. For the LDF measurements, a new cohort of animals was subjected to the same injury/treatment; however, these rats were not tested for oxidative stress state, apoptosis and newborn neurons.

\section{Morris water maze}

Cognitive function was measured by Morris water maze (MWM) (Huaibeizhenghua, China) as previously described by Tian [23]. Briefly, at 14 days post-injury, some rats were subjected to a hidden platform trial three times a day for four days, and the escape latency was recorded. At $18 \mathrm{~d}$ post-injury, the probe trial was performed to record the crossover number of the rats. A video camera was fixed to the ceiling of the MWM device and connected to the computer with an automated tracking system to collect the data, and the data were 
analyzed using the Animal Behavior Video Analysis System (Huaibeizhenghua, China). The MWM measurements were performed on the second cohort of animals subject to the same injury/treatment, although oxidative stress state, apoptosis and newborn neurons were not measured in this cohort.

Detection of malondialdehyde and superoxide dismutase levels

Oxidative stress was assessed by serum MDA and SOD levels. At one, three, seven and $14 \mathrm{~d}$ post-injury, $1 \mathrm{ml}$ of blood was collected from the tail vein of rats. The levels of MDA and SOD in the serum of rats were detected by MDA and SOD kits (Beyotime Biotechnology, China) according to the manufacturer's instructions.

\section{Brain coronal slices preparation}

At $14 \mathrm{~d}$ post-injury, 12 rats of each group were anesthetized with $2 \%$ pentobarbital sodium $(0.30 \mathrm{ml} / 100 \mathrm{~g})$, and the brains were removed after perfusion and fixed with $4 \%$ paraformaldehyde at room temperature for 4 hours. The $15 \mu \mathrm{m}$-thick brain coronal slices $(-2.5$ to $-3.5 \mathrm{~mm}$ from the bregma) was prepared using a cryostat (Leica CM1900) and each slice was attached to a glass slide.

\section{Apoptosis was detected by TUNEL}

The apoptotic cells of cortex were detected by the TUNEL Kit (Millipore, USA). Three brain coronal sections (front, middle and rear injury zone) in each rat were analysed. The brain coronal slices were blocked with $10 \%$ goat serum in $0.01 \mathrm{M}$ sodium phosphate buffer (containing $0.05 \% \mathrm{v} / \mathrm{v}$ Tween $20, \mathrm{pH} 7.4$ ) for $1 \mathrm{~h}$ at room temperature. Each slice was then incubated with $50 \mu \mathrm{l}$ TUNEL test solution for $2 \mathrm{~h}$ at room temperature and stained with Hoechst33342 for $0.5 \mathrm{~h}$ at RT to visualize cell nuclei. The sections were observed under a fluorescent microscope (Leica, German). The positive cells were counted manually under 400 times microscope at 10 random view fields in the injured area of each slice by an analyst who was blinded to the experimental group for each slice.

\section{Immunofluorescence}

Three brain coronal sections (front, middle and rear injury zone) in each rat were analysed. Brain coronal slices were blocked with $10 \%$ goat serum in $0.01 \mathrm{M}$ sodium phosphate buffer (containing $0.05 \%$ (v/v) Tween $20, \mathrm{pH} 7.4$ ) for $1 \mathrm{~h}$ at room temperature. The slices were incubated with rat anti-BrdU (1:200, Abcam), guinea pig anti-DCX (neuronal migration protein doublecortin, DCX) (1:800, Millipore), mouse monoclonal anti-NeuN (hexaribonucleotide binding protein-3, NeuN) (1:500, Millipore) overnight at $4^{\circ} \mathrm{C}$, followed by incubation with Alexa Fluor 568-conjugated goat anti-rat IgG (1:800, Molecu- lar Probes, USA), 488-conjugated goat anti- guinea pig or mouse IgG (1:200, Invitrogen, USA). Cell nuclei were stained with Hoechst33342 for $0.5 \mathrm{~h}$ at RT. The sections were observed under a fluorescent microscope (Leica, German). The positive cells (stained by green, red and blue together) were counted manually under 400 times microscope at 10 random view fields in the injured area of each slice by the analyst who was blinded to the experimental groups.

\section{Statistical analysis}

The statistics package for social science 21.0 (SPSS 21.0, IBM, USA) was applied to analyze the data in this study. The measured data in this study were in accordance with the normal distribution and are expressed as mean $\pm S D$. Statistical comparisons were performed using one-way analysis of variance with the Bonferroni test. Differences of $P<0.05$ were considered statistically significant.

\section{RESULTS}

\section{Cerebral blood flow levels after traumatic brain injury}

Compared with sham group, the CBF levels in the TBI+vehicle group decreased at one, 12, 24 and $48 \mathrm{~h}$ post-injury $(P<0.05)$, and the CBF level in the TBI+melatonin group significantly decreased at 24 and $48 \mathrm{~h}$ post-injury $(P<0.05)$. The $\mathrm{CBF}$ level in the TBI+melatonin group was higher than the $\mathrm{TBI}+$ vehicle group at the corresponding time point, but the difference was not statistically significant $(P>0.05)$ (Figure $1 A)$.

\section{Cognitive function}

The cognitive function of the rats was measured by MWM. Compared with the sham group, the escape latency of the rats in the $\mathrm{TBI}+$ vehicle and $\mathrm{TBI}+$ melatonin groups was significantly prolonged $(P<0.05)$, but the escape latency of the $\mathrm{TBI}+$ melatonin group was less than that of the $\mathrm{TBI}+$ vehicle group (Figure 1B). Compared with the sham group, the platform crossover number of the rats in the $\mathrm{TBI}+$ vehicle and $\mathrm{TBI}+$ melatonin groups was significantly reduced $(P<0.05)$, but the platform crossover number of the $\mathrm{TBI}+$ melatonin group was more than that of the $\mathrm{TBI}+$ vehicle group (Figure 1C).

Oxidative stress level was assessed with serum malondialdehyde and superoxide dismutase levels

Compared with sham group, the MDA level in the TBI+vehicle and $\mathrm{TBI}+$ melatonin groups increased significantly at one, three, seven and $14 \mathrm{~d}$ post-injury $(P<0.05)$. The MDA level in the TBI+melatonin group was lower than the TBI+vehicle group $(P<0.05)$ and it was close to the sham group at $14 \mathrm{~d}(P>0.05)$ (Figure $2 A)$. Compared with the sham group, the SOD level in the TBI+vehicle and 
FIGURE 1.

CEREBRAL BLOOD FLOW AND COGNITIVE FUNCTION OF RATS WAS MEASURED BY LASER DOPPLER FLOWMETRY AND THE MORRIS WATER MAZE ASSAY.
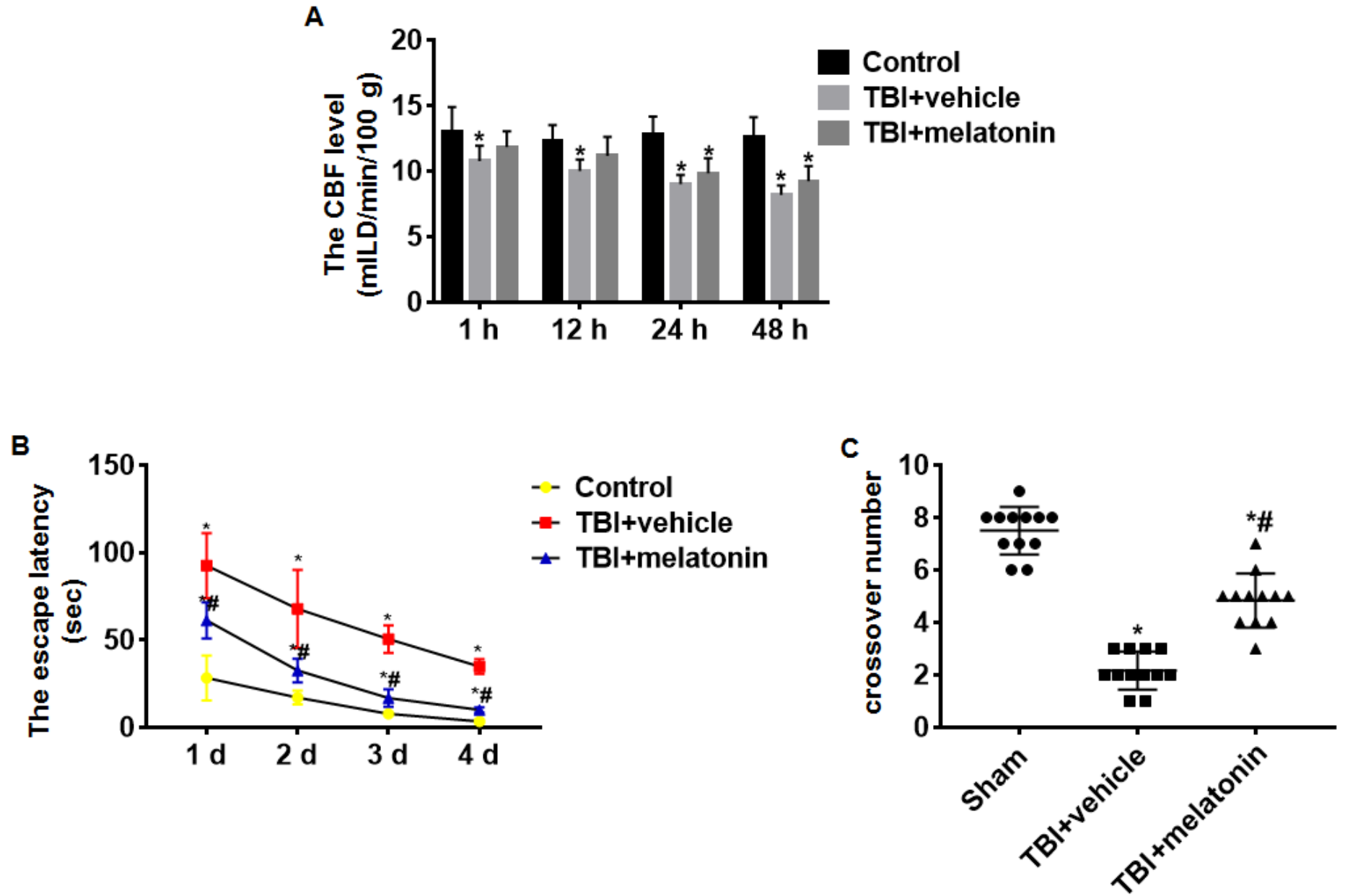

(A) Compared with sham group, the CBF level in the TBI+vehicle decreased significantly at one, 12, 24 and $48 \mathrm{~h}$ post-injury, the CBF level in the TBI+melatonin group decreased significantly at 24 and $48 \mathrm{~h}$ post-injury. The CBF level in the TBl+melatonin group was higher than that of the TBl+vehicle group at corresponding time points. (B) Compared with the sham group, the escape latency of the rats in the TBI+vehicle and TBI+melatonin groups was significantly prolonged, but the escape latency of the TBI+melatonin group was less than that of the TBI+vehicle group. (C) Compared with the sham group, the platform crossover number of the rats in the TBI+vehicle and TBI+melatonin groups was significantly reduced, but the platform crossover number of the TBI+melatonin group was more than that of the TBI+vehicle group. ${ }^{*} P<0.05$ vs. sham group. \#P<0.05 vs. $T B I+v e h i c l e ~ g r o u p$. Error bars indicate $S D, n=12$ in all three groups.

TBI+melatonin groups decreased significantly at one, three, seven and $14 \mathrm{~d}$ post-injury, and the SOD level in the $\mathrm{TBI}+$ melatonin group was higher than the TBI+vehicle group $(P<0.05)$ and it was close to the sham group at $14 \mathrm{~d}(P>0.05)$ (Figure 2B).

Apoptosis was detected by the terminal deoxynucleotidyl transferase dUTP nick end labelling assay

The apoptotic cells were detected by the TUNEL assay. Few apoptotic cells were found in cortex of the sham group. A few apoptotic cells were found in the injured cortex of the $\mathrm{TBI}+$ melatonin group and more apoptotic cells were found in injured cortex of the TBI+vehicle group, and the difference among the groups was statistically significant $(P<0.05)$ (Figure 3 ).

Newborn immature neurons in the injured cortex

Newborn immature neurons were detected by DCX/ BrdU immunofluorescence staining. Few $\mathrm{DCX}^{+} / \mathrm{BrdU}^{+}$ cells were found in the cortex of the sham group. A few $\mathrm{DCX}^{+} / \mathrm{BrdU}^{+}$cells were found in the injured cortex of the $\mathrm{TBI}+$ vehicle group and more $\mathrm{DCX}^{+} / \mathrm{BrdU}^{+}$cells were found in the injured cortex of the TBI+melatonin group, and the difference among groups was statistically significant $(P<0.05)$ (Figure 4). 
FIGURE 2.

\section{OXIDATIVE STRESS IN RATS WAS ASSESSED BY SERUM} MDA AND SOD LEVELS.
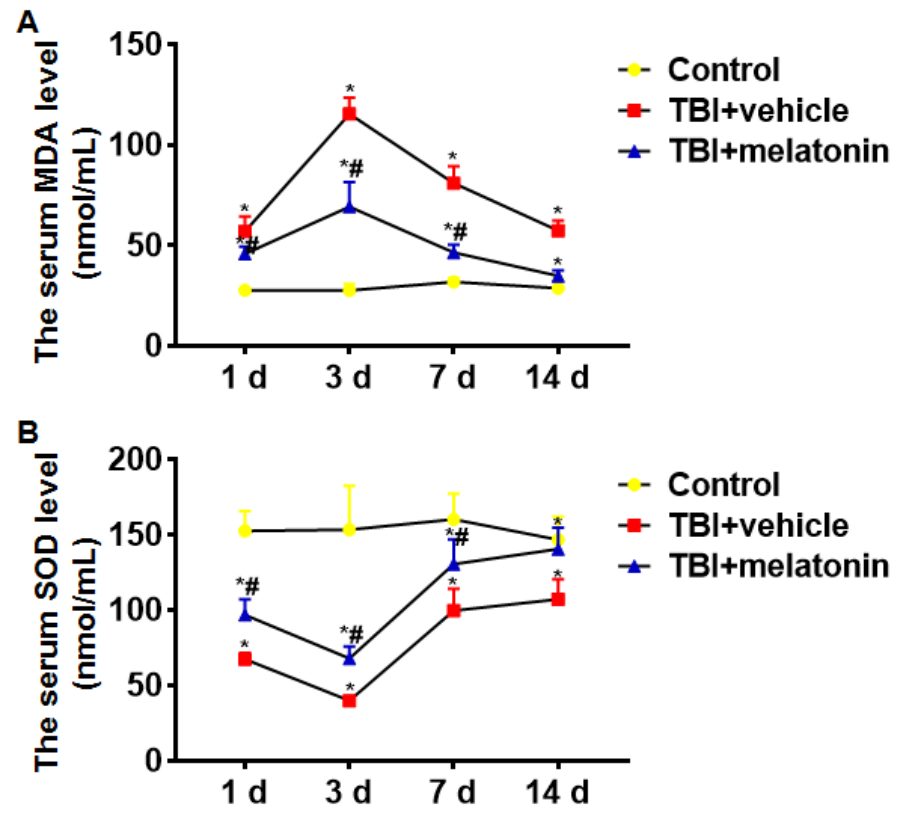

(A) Compared with sham group, the MDA levels in the TBI+vehicle and TBI+melatonin groups increased significantly at one, three, seven and $14 d$ post-injury. The MDA level in the TBI+melatonin group was lower than that of the TBI+vehicle group and it was close to the sham group at $14 \mathrm{~d}$. (B) Compared with the sham group, the SOD level in the TBI+vehicle and TBI+melatonin groups decreased significantly at one, three, seven, $14 \mathrm{~d}$ post-injury. The SOD level in the TBI+melatonin group was higher than that of the TBI+vehicle group and it was close to the sham group at $14 d$. ${ }^{*} P<$ 0.05 vs. sham group. \#P<0.05 vs. TBI+vehicle group. Error bars indicate SD, $n=6$ in all three groups.
FIGURE 3.

\section{CELL APOPTOSIS WAS DETECTED BY TERMINAL DEOXYNUCLEOTIDYL TRANSFERASE DUTP NICK END LABELLING (TUNEL) ASSAY.}
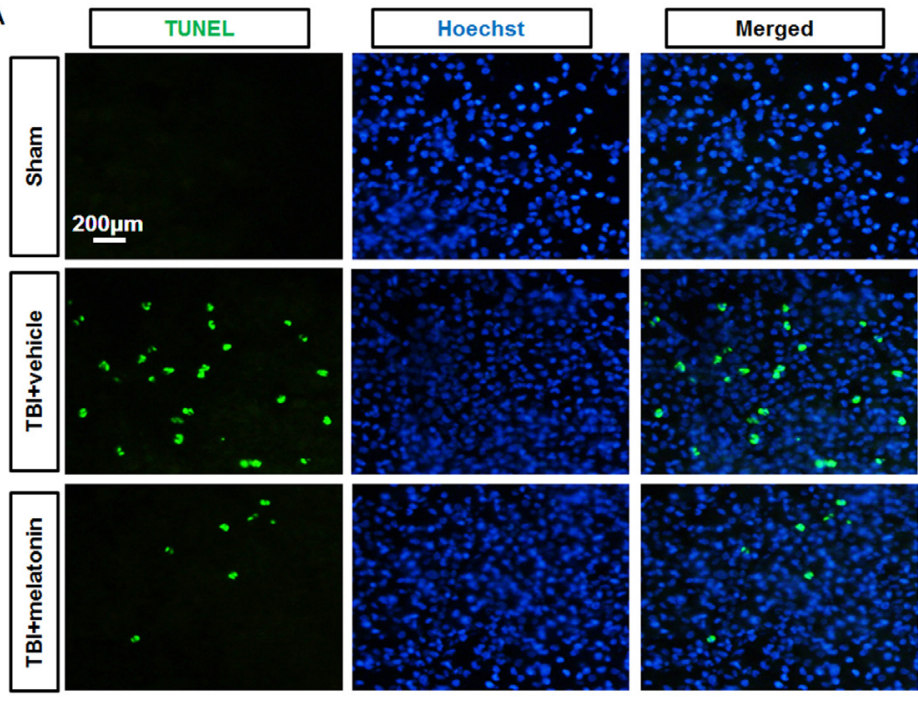

B

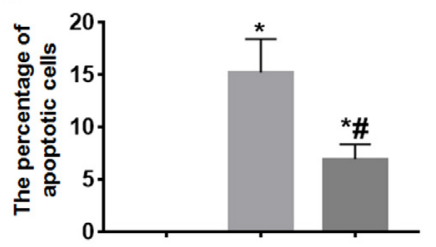

Sham

TB|+vehicle

- TBI+melatonin

(A) Apoptotic cells were detected by the TUNEL assay. Few apoptotic cells were found in cortex of the sham group. A few apoptotic cells (shown by the arrows) were found in injured cortex of the TBI+melatonin group. More apoptotic cells (shown by the arrows) were found in injured cortex of the TBI+vehicle group. (B) Statistical graph of percentage of apoptotic cells. ${ }^{*} P<0.05$ vs. sham group. \#P<0.05 vs. TBI+vehicle group. Error bars indicate $S D, n=12$ in all three groups.

Newborn mature neurons in the injured cortex

Newborn mature neurons were detected by NeuN/BrdU immunofluorescence staining. Few $\mathrm{NeuN}^{+} / \mathrm{BrdU}^{+}$cells were found in cortex of the sham group, a few $\mathrm{NeuN}^{+} /$ $\mathrm{BrdU}^{+}$cells were found in cortex of the TBI+vehicle group and more $\mathrm{NeuN}^{+} / \mathrm{BrdU}^{+}$cells were found in the injured cortex of the TBI+melatonin group. The difference among groups was statistically significant $(P<0.05)$ (Figure 5).

\section{DISCUSSION}

Initial traumatic brain injuries include skull fractures, acute cerebrovascular injury (in the acute phase) and mechanical damage to brain tissue. These events lead to a series of cellular and molecular responses that cause secondary brain damage. These initial traumatic events also lead to chronic hypoperfusion in the cerebral cortex, which results in the poor delivery of oxygen and metabolites, and aggravates the secondary brain damage and leads to poor cognitive outcomes
[21]. Secondary brain injury primarily involves highly reactive species, such as free radicals, reactive oxygen species and reactive nitrogen species. These reactants can damage the cell's DNA and protein structure, leading to oxidative stress $[24,25]$. All these lead to neuronal loss in TBI [9], which plays a major role in brain injury. In this study, compared with sham group, the CBF level in TBI rats decreased at one, 12, 24 and $48 \mathrm{~h}$ post-injury, the serum MDA level increased and the serum SOD level decreased in TBI rats at one, three, seven and 14 $\mathrm{d}$ post-injury, which indicated that TBI rats were in the state of hypoperfusion and oxidative stress. The results of MWM showed the learning and memory functions of TBI rats decreased.

With the lack of effective therapy to repair injured brain tissue, it is of the utmost importance to develop novel and effective therapies. Melatonin is a lipophilic neurohormone secreted primarily from the pineal gland. Melatonin freely crosses the blood-brain barrier [26] 


\section{FIGURE 4.}

NEWBORN IMMATURE NEURONS IN THE INJURED CORTEX.

A
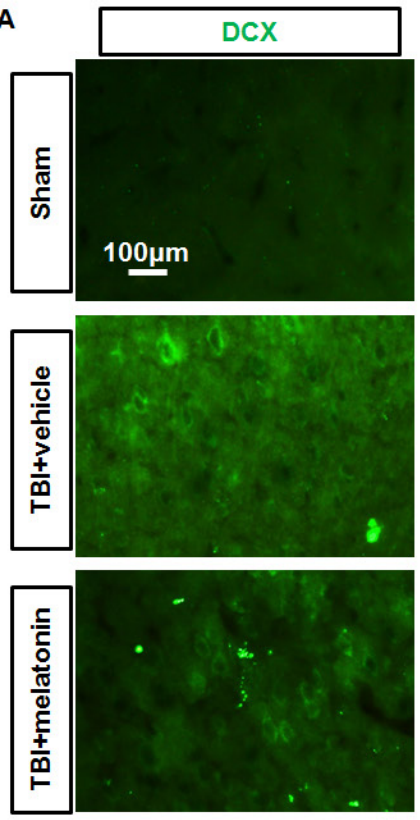
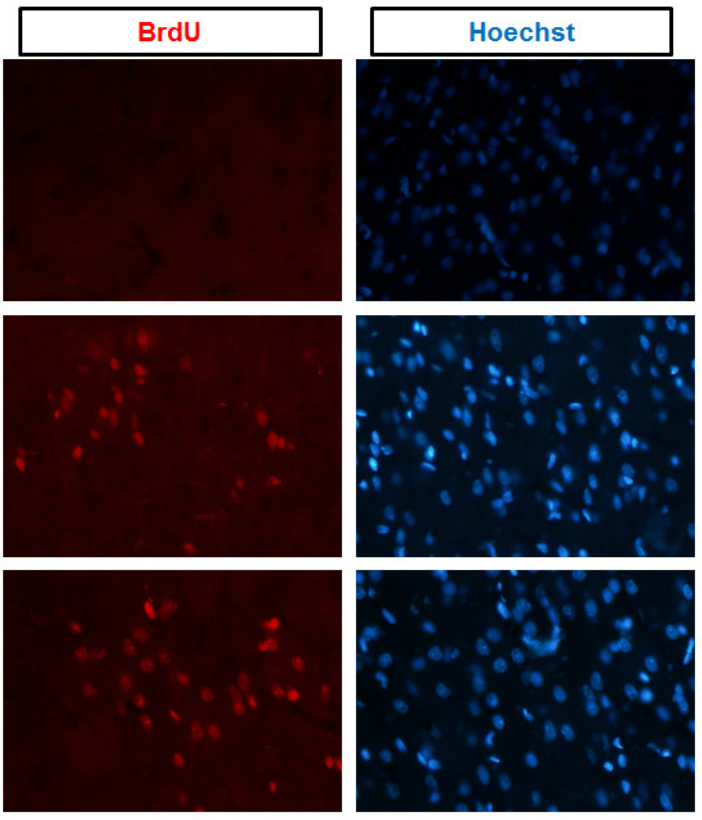
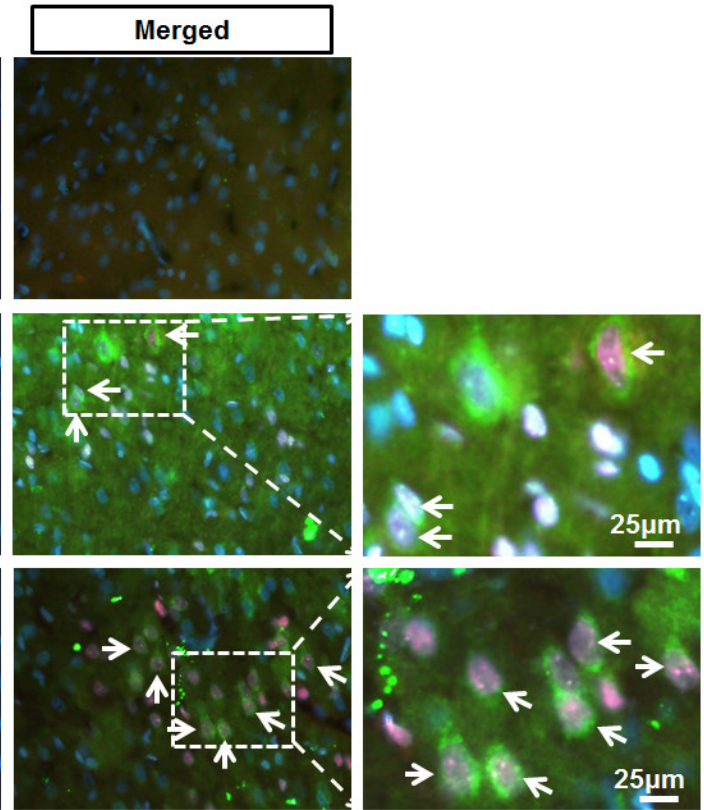

B

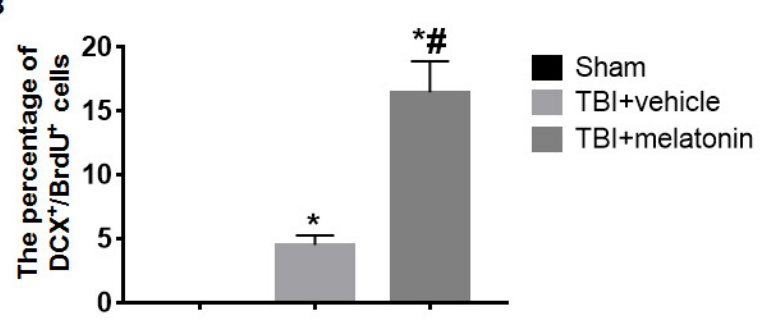

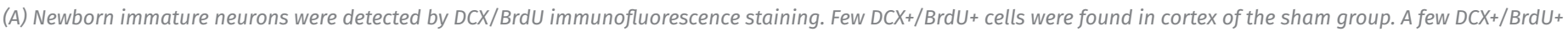

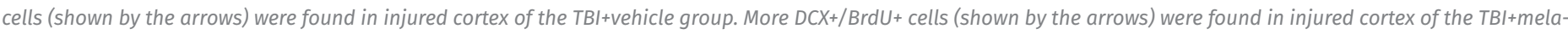

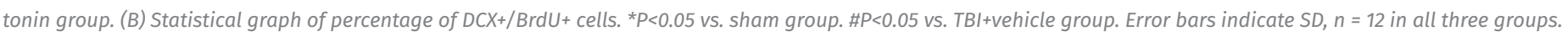

and this ability allows for the application of melatonin in the treatment of central nervous system diseases. A number of studies have documented melatonin's various physiological functions in the body, such as modulation of human mood and behavior [27], anticancer activity [28,29], sleep regulation [30], anti-inflammatory properties [31] and free radical scavenging [32]. Previous studies showed that melatonin has a potential neuroprotective effect following TBI [33-35]. It has been reported that melatonin protected against brain injury by reducing inflammation [31], edema [36] and oxidative damage [36], and by regulating the blood-brain barrier integrity, the expression of NF- $\beta$ [37].

It is unknown whether there is a sex difference in TBI and melatonin effect; therefore, to eliminate the possible influence of sex differences on these results, we designed the experimental groups to have the same sex composition, so that the data obtained in each group were comparable. Oxidative stress level was assessed by measuring MDA and SOD levels, and the results showed that melatonin significantly reduced oxidative stress in TBI rats. The TUNEL detection in this study showed that melatonin protected cells from apoptosis, which was consistent with previous studies. The proposed protective mechanisms of melatonin are multiple, and include both anti-excitotoxic [38] and antioxidative properties $[39,40]$. In this study, we also found that melatonin improved the CBF to some extent after TBI, which may be related with the melatonin receptors (MT1, MT2) in vascular smooth muscle [41]; however, the mechanism involved is still unclear. The optimal timing of melatonin treatment and possible sex difference in melatonin effect are yet to be determined.

Whether neurogenesis is induced in the cortex after TBI is still controversial, although adult neurogenesis occurs in the subventricular zone of the ventricles and 
FIGURE 5.

NEWBORN MATURE NEURONS IN THE INJURED CORTEX.
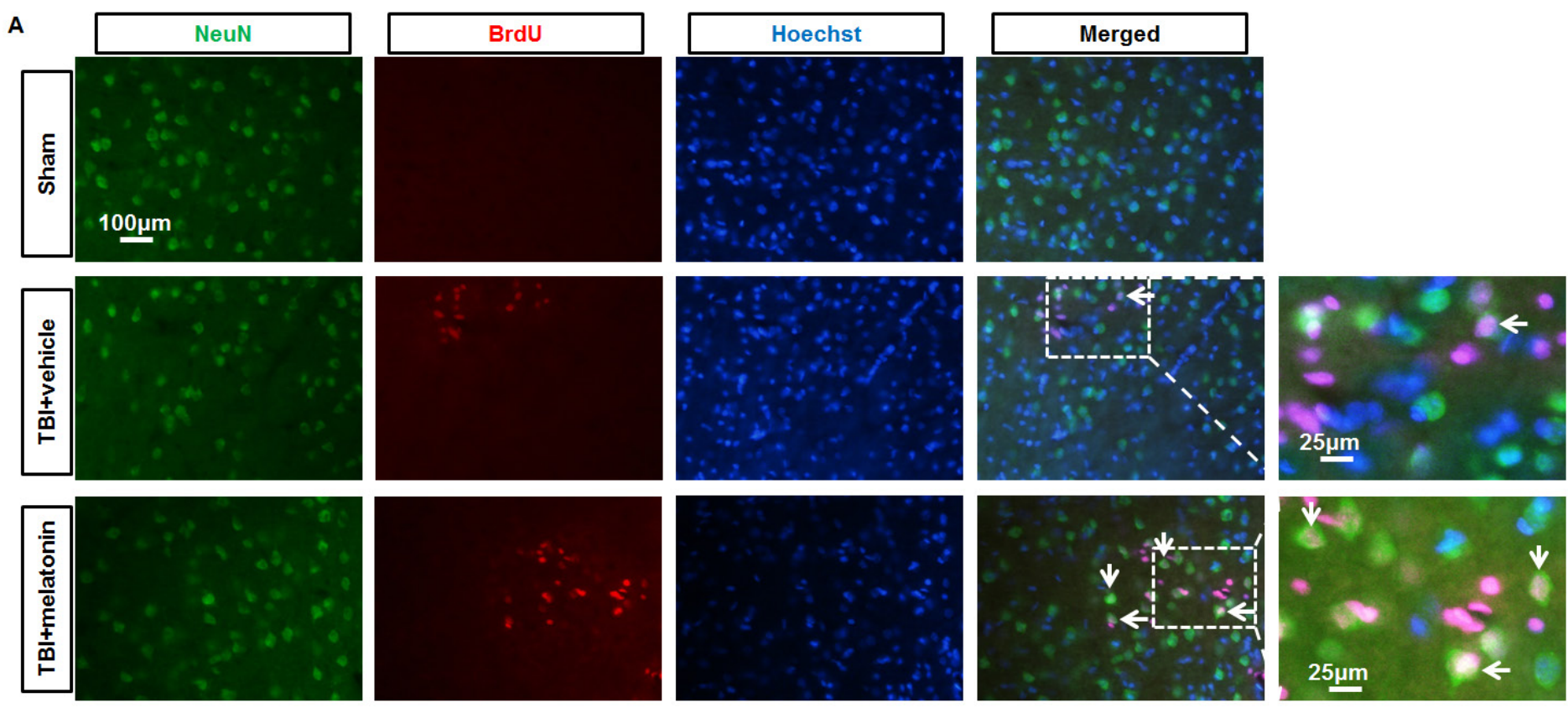

B

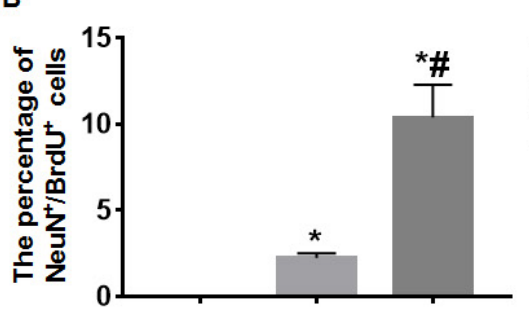

Sham

TBI+vehicle

$\mathrm{TB} \mid+$ melatonin

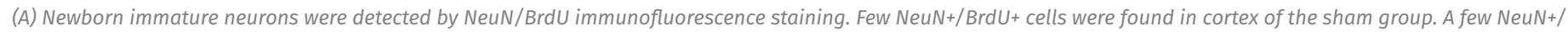

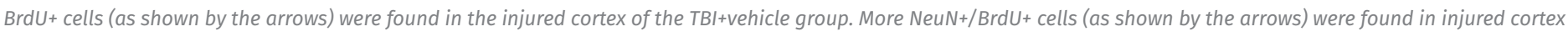

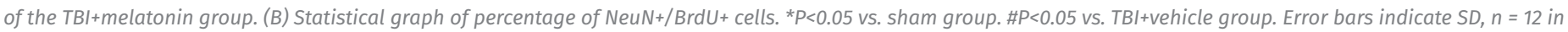
all three groups.

in the subgranular zone of the dentate gyrus of the hippocampus [42]. Previous studies showed that brain injury can stimulate proliferation of endogenous NSCS but the activated NSCS failed to become mature neurons in the injured cortex $[9,43,44]$ : perhaps these cells did not survive due to apoptosis [45]. In this study, newborn immature and mature neurons were detected by DCX/ BrdU and NeuN/BrdU staining, respectively. The results showed newborn immature emerged in the injured cortex but newborn mature neurons were found only in the $\mathrm{TBI}+$ melatonin group. This observation indicates that neurogenesis emerged in the injured cortex and melatonin promoted the differentiation of neuron in the injured cortex after TBI. It has been demonstrated previously that melatonin is involved in cytoskeleton organization by inducing microtubule polymerization through affecting the function of some microtubule-associated proteins [46]. The effects of melatonin on the differentiation of neurons in the injured cortex might be related to the function of the indole ring of the hormone that regulates cytoskeletal organization, and cytoskeleton organization may be related to dendrite maturation of immature neurons and possibly to neuron migration, and antioxidant and anti-apoptotic capacities related to survival [47]. However, neurogenesis after TBI may produce both beneficial (functional recovery) and pathological (epilepsy) changes. To observe the effect of neurogenesis after TBI, in this study we used MWM to evaluate the cognitive function of the rats. The results showed melatonin could significantly improve the learning and memory functions of TBI rats. Whether neurogenesis after TBI could cause pathological changes needs further research.

The results of the current study suggest that melatonin reduces oxidative stress level and cell apoptosis of 
brain, promotes the regeneration of neurons in injury cortex and thus improves the cognitive functional recovery of the rats with TBI.

\section{FINANCIAL SUPPORT}

This work was supported by National Natural Science Foundation of China (No. 81801301), the Key Talents Project for Youth Medicine in Jiangsu Province (QNRC2016397), and the Jiangsu Pharmaceutical Association-Aosaikang Clinical Pharmacy Fund (A201819).

\section{REFERENCES}

1. Beauchamp K, Mutlak H, Smith WR, Shohami E, Stahel PF. Pharmacology of traumatic brain injury: where is the "golden bullet"? Molecular medicine. Nov-Dec 2008;14(1112):731-40. doi:10.2119/2008-00050.Beauchamp

2. Leker RR, Shohami E. Cerebral ischemia and trauma-different etiologies yet similar mechanisms: neuroprotective opportunities. Brain research Brain research reviews. Jun 2002;39(1):55-73.

3. Gruenbaum SE, Zlotnik A, Gruenbaum BF, Hersey D, Bilotta F. Pharmacologic Neuroprotection for Functional Outcomes After Traumatic Brain Injury: A Systematic Review of the Clinical Literature. CNS drugs. Jun 23 2016;doi:10.1007/ s40263-016-0355-2

4. Kinoshita K. Traumatic brain injury: pathophysiology for neurocritical care. Journal of intensive care. 2016;4:29. doi:10.1186/s40560-016-0138-3

5. Wee HY, Lim SW, Chio CC, Niu KC, Wang CC, Kuo JR. Hyperbaric oxygen effects on neuronal apoptosis associations in a traumatic brain injury rat model. The Journal of surgical research. Aug 2015;197(2):382-9. doi:10.1016/j.jss.2015.04.052

6. Naziroglu M, Senol N, Ghazizadeh V, Yuruker V. Neuroprotection induced by $\mathrm{N}$-acetylcysteine and selenium against traumatic brain injury-induced apoptosis and calcium entry in hippocampus of rat. Cellular and molecular neurobiology. Aug 2014;34(6):895-903. doi:10.1007/s10571014-0069-2

7. Northington FJ, Ferriero DM, Graham EM, Traystman RJ, Martin LJ. Early Neurodegeneration after Hypoxia-Ischemia in Neonatal Rat Is Necrosis while Delayed Neuronal Death Is Apoptosis. Neurobiol Dis. Apr 2001;8(2):207-19. doi:10.1006/nbdi.2000.0371

8. Hilario E, Rey-Santano MC, Goni-de-Cerio F, et al. Cerebral blood flow and morphological changes after hypoxic-ischaemic injury in preterm lambs. Acta Paediatr. Jul 2005;94(7):903-11. doi:10.1080/08035250510031151

9. Yi X, Jin G, Zhang X, et al. Cortical endogenic neural regeneration of adult rat after traumatic brain injury. PloS one. 2013;8(7):e70306. doi:10.1371/journal.pone.0070306

10. Ugoya SO, Tu J. Bench to bedside of neural stem cell in traumatic brain injury. Stem cells international. 2012;2012:141624. doi:10.1155/2012/141624
11. Rosen J, Than NN, Koch D, Poeggeler B, Laatsch H, Hardeland R. Interactions of melatonin and its metabolites with the ABTS cation radical: extension of the radical scavenger cascade and formation of a novel class of oxidation products, C2-substituted 3-indolinones. Journal of pineal research. Nov 2006;41(4):374-81. doi:10.1111/j.1600079X.2006.00379.x

12. Tan DX, Manchester LC, Terron MP, Flores LJ, Reiter RJ. One molecule, many derivatives: a never-ending interaction of melatonin with reactive oxygen and nitrogen species? Journal of pineal research. Jan 2007;42(1):28-42. doi:10.1111/ j.1600-079X.2006.00407.x

13. Rodriguez C, Mayo JC, Sainz RM, et al. Regulation of antioxidant enzymes: a significant role for melatonin. Journal of pineal research. Jan 2004;36(1):1-9.

14. Tomas-Zapico C, Coto-Montes A. A proposed mechanism to explain the stimulatory effect of melatonin on antioxidative enzymes. Journal of pineal research. Sep 2005;39(2):99104. doi:10.1111/j.1600-079X.2005.00248.x

15. Carloni S, Perrone S, Buonocore G, Longini M, Proietti $\mathrm{F}$, Balduini W. Melatonin protects from the long-term consequences of a neonatal hypoxic-ischemic brain injury in rats. Journal of pineal research. Mar 2008;44(2):157-64. doi:10.1111/j.1600-079X.2007.00503.x

16. Alonso-Alconada D, Alvarez A, Lacalle J, Hilario E. Histological study of the protective effect of melatonin on neural cells after neonatal hypoxia-ischemia. Histol Histopathol. Jun 2012;27(6):771-83.

17. Barlow KM, Esser MJ, Veidt M, Boyd R. Melatonin as a Treatment after Traumatic Brain Injury: A Systematic Review and Meta-Analysis of the Pre-Clinical and Clinical Literature. Journal of neurotrauma. Feb 2019;36(4):523-537. doi:10.1089/ neu.2018.5752

18. Osier N, McGreevy E, Pham L, et al. Melatonin as a Therapy for Traumatic Brain Injury: A Review of Published Evidence. International journal of molecular sciences. May 2018;19(5) doi:10.3390/ljms19051539

19. Salman M, Kaushik P, Tabassum H, Parvez S. Melatonin Provides Neuroprotection Following Traumatic Brain Injury-Promoted Mitochondrial Perturbation in Wistar Rat. Cellular and molecular neurobiology. May 28 2020;doi:10.1007/ s10571-020-00884-5

20. Ozdemir D, Uysal N, Gonenc S, et al. Effect of melatonin on brain oxidative damage induced by traumatic brain injury in immature rats. Physiol Res. 2005;54(6):631-637.

21. Rafols JA, Kreipke CW, Petrov T. Alterations in cerebral cortex microvessels and the microcirculation in a rat model of traumatic brain injury: a correlative EM and laser Doppler flowmetry study. Neurological research. Jun 2007;29(4):33947. doi:10.1179/016164107X204648

22. Takahashi A, Park HK, Melgar MA, et al. Cerebral cortex blood flow and vascular smooth muscle contractility in a rat model of ischemia: a correlative laser Doppler flowmetric and scanning electron microscopic study. Acta neuropathologica. Apr 1997;93(4):354-68. doi:10.1007/ s004010050627 
23. Tian H, Ding N, Guo M, et al. Analysis of Learning and Memory Ability in an Alzheimer's Disease Mouse Model using the Morris Water Maze. Journal of visualized experiments : JOVE. Oct 29 2019;(152)doi:10.3791/60055

24. Bains M, Hall ED. Antioxidant therapies in traumatic brain and spinal cord injury. Biochimica et biophysica acta. May 2012;1822(5):675-84. doi:10.1016/j.bbadis.2011.10.017

25. Khalatbary AR, Tiraihi T, Boroujeni MB, Ahmadvand $H$, Tavafi M, Tamjidipoor A. Effects of epigallocatechin gallate on tissue protection and functional recovery after contusive spinal cord injury in rats. Brain research. Jan 8 2010;1306:168-75. doi:10.1016/j.brainres.2009.09.109

26. Tarocco A, Caroccia N, Morciano G, et al. Melatonin as a master regulator of cell death and inflammation: molecular mechanisms and clinical implications for newborn care. Cell death \& disease. Apr 8 2019;10(4):317. doi:10.1038/ s41419-019-1556-7

27. Naseem M, Parvez S. Role of melatonin in traumatic brain injury and spinal cord injury. TheScientificWorldJournal. 2014;2014:586270. doi:10.1155/2014/586270

28. McCarty MF. Minimizing the cancer-promotional activity of cox-2 as a central strategy in cancer prevention. Medical hypotheses. Jan 2012;78(1):45-57. doi:10.1016/j. mehy.2011.09.039

29. Santoro R, Marani M, Blandino G, Muti P, Strano S. Melatonin triggers p53Ser phosphorylation and prevents DNA damage accumulation. Oncogene. Jun 14 2012;31(24):293142. doi:10.1038/onc.2011.469

30. Cardinali DP, Srinivasan V, Brzezinski A, Brown GM. Melatonin and its analogs in insomnia and depression. Journal of pineal research. May 2012;52(4):365-75. doi:10.1111/j.1600079X.2011.00962.x

31. Esposito E, Cuzzocrea S. Antiinflammatory activity of melatonin in central nervous system. Current neuropharmacology. Sep 2010;8(3):228-42. doi:10.2174/157015910792246155

32. Galano A, Tan DX, Reiter RJ. Melatonin as a natural ally against oxidative stress: a physicochemical examination. Journal of pineal research. Aug 2011;51(1):1-16. doi:10.1111/ j.1600-079X.2011.00916.x

33. Barlow KM, Esser MJ, Veidt M, Boyd R. Melatonin as a Treatment after Traumatic Brain Injury: A Systematic Review and Meta-Analysis of the Pre-Clinical and Clinical Literature. Journal of neurotrauma. Feb 15 2019;36(4):523537. doi:10.1089/neu.2018.5752

34. Grima NA, Rajaratnam SMW, Mansfield D, Sletten TL, Spitz G, Ponsford JL. Efficacy of melatonin for sleep disturbance following traumatic brain injury: a randomised controlled trial. BMC medicine. Jan 19 2018;16(1):8. doi:10.1186/s12916017-0995-1

35. Osier N, McGreevy E, Pham L, et al. Melatonin as a Therapy for Traumatic Brain Injury: A Review of Published Evidence. International journal of molecular sciences. May 22 2018;19(5)doi:10.3390/ijms19051539
36. Dehghan F, Khaksari Hadad M, Asadikram G, Najafipour H, Shahrokhi N. Effect of melatonin on intracranial pressure and brain edema following traumatic brain injury: role of oxidative stresses. Archives of medical research. May 2013;44(4):251-8. doi:10.1016/j.arcmed.2013.04.002

37. Beni SM, Kohen R, Reiter RJ, Tan DX, Shohami E. Melatonin-induced neuroprotection after closed head injury is associated with increased brain antioxidants and attenuated late-phase activation of NF-kappaB and AP-1. FASEB journal : official publication of the Federation of American Societies for Experimental Biology. Jan 2004;18(1):149-51. doi:10.1096/fj.03-0323fje

38. Gressens P, Schwendimann L, Husson I, et al. Agomelatine, a melatonin receptor agonist with $5-\mathrm{HT}(2 \mathrm{C})$ receptor antagonist properties, protects the developing murine white matter against excitotoxicity. Eur J Pharmacol. Jun 24 2008;588(1):58-63. doi:10.1016/j.ejphar.2008.04.016

39. Gitto E, Pellegrino S, Gitto P, Barberi I, Reiter RJ. Oxidative stress of the newborn in the pre- and postnatal period and the clinical utility of melatonin. Journal of pineal research. Mar 2009;46(2):128-39. doi:10.1111/j.1600-079X.2008.00649.x

40. Balduini W, Carloni S, Perrone S, et al. The use of melatonin in hypoxic-ischemic brain damage: an experimental study. J Matern Fetal Neonatal Med. Apr 2012;25 Suppl 1:11924. doi:10.3109/14767058.2012.663232

41. Doolen S, Krause DN, Dubocovich ML, Duckles SP. Melatonin mediates two distinct responses in vascular smooth muscle. European Journal of Pharmacology. 1998;345(1):67.

42. Lie DC, Colamarino SA, Song HJ, et al. Wnt signalling regulates adult hippocampal neurogenesis. Nature. Oct 27 2005;437(7063):1370-5. doi:10.1038/nature04108

43. Brazel CY, Nunez JL, Yang Z, Levison SW. Glutamate enhances survival and proliferation of neural progenitors derived from the subventricular zone. Neuroscience. 2005;131(1):5565. doi:10.1016/j.neuroscience.2004.10.038

44. Ikeda T, Iwai M, Hayashi T, et al. Limited differentiation to neurons and astroglia from neural stem cells in the cortex and striatum after ischemia/hypoxia in the neonatal rat brain. Am J Obstet Gynecol. Sep 2005;193(3 Pt 1):849-56. doi:10.1016/j.ajog.2005.01.029

45. Otero L, Zurita M, Bonilla C, et al. Endogenous neurogenesis after intracerebral hemorrhage. Histol Histopathol. Mar 2012;27(3):303-15.

46. Bellon A, Ortiz-Lopez L, Ramirez-Rodriguez G, Anton-Tay $\mathrm{F}$, Benitez-King $\mathrm{G}$. Melatonin induces neuritogenesis at early stages in N1E-115 cells through actin rearrangements via activation of protein kinase $\mathrm{C}$ and Rho-associated kinase. Journal of pineal research. Apr 2007;42(3):214-21. doi:10.1111/j.1600-079X.2006.00408.x

47. Benitez-King G. Melatonin as a cytoskeletal modulator: implications for cell physiology and disease. Journal of pineal research. Jan 2006;40(1):1-9. doi:10.1111/j.1600079X.2005.00282.x 